\title{
Perceived Stress and Adherence to the Dietary Recommendations and Blood Glucose Levels in Type 1 Diabetes
}

\author{
Aila J. Ahola $\mathbb{D}^{1,2,3}$ Carol Forsblom $\mathbb{D}^{1,2,3}$ Valma Harjutsalo ${ }^{1},{ }^{1,2,3,4}$ \\ and Per-Henrik Groop iD $1,2,3,5$ \\ ${ }^{1}$ Folkhälsan Institute of Genetics, Folkhälsan Research Center, Helsinki, Finland \\ ${ }^{2}$ Abdominal Center, Nephrology, University of Helsinki and Helsinki University Central Hospital, Helsinki, Finland \\ ${ }^{3}$ Research Program for Clinical and Molecular Metabolism, Faculty of Medicine, University of Helsinki, Finland \\ ${ }^{4}$ Chronic Disease Prevention Unit, National Institute for Health and Welfare, Helsinki, Finland \\ ${ }^{5}$ Department of Diabetes, Central Clinical School, Monash University, Melbourne, Victoria, Australia
}

Correspondence should be addressed to Per-Henrik Groop; per-henrik.groop@helsinki.fi

Received 2 March 2020; Accepted 20 June 2020; Published 18 July 2020

Academic Editor: Pedro M. Geraldes

Copyright (c) 2020 Aila J. Ahola et al. This is an open access article distributed under the Creative Commons Attribution License, which permits unrestricted use, distribution, and reproduction in any medium, provided the original work is properly cited.

\begin{abstract}
Stress may negatively impact self-management of diabetes and thereby deteriorate glycaemic control. Eating is the most frequently reported stress-release method. In this study, we investigated the association between perceived stress (PS), dietary adherence, and glycaemic control. Data from participants in the FinnDiane Study with type 1 diabetes who had completed a diet questionnaire and Cohen's perceived stress scale (PSS) were included. In addition to using a continuous PSS score, participants were divided into three groups based on the PSS scores: the first PSS quartile, low levels of PS; second and third quartiles, moderate levels of PS; and fourth quartile, high levels of PS. A diet score reflecting the level of adherence to dietary recommendations was calculated. Analyses were conducted in the whole sample and in subgroups divided by body mass index (BMI $<25 \mathrm{~kg} / \mathrm{m}^{2} \mathrm{vs}$. BMI $\geq 25 \mathrm{~kg} / \mathrm{m}^{2}$ ). In the whole sample, high PS and continuous stress score were negatively associated with the diet score and with adherence to fish, fresh vegetable, low-fat liquid milk product, and vegetable oil-based cooking fat recommendations. The stress score was negatively associated with the diet score both in lean and in those overweight or obese. However, fish and fresh vegetable recommendations were only affected in those with corpulence. PS was not associated with mean blood glucose concentrations in the whole sample. When divided by BMI status, worse glycaemic control was observed in lean subjects reporting stress. In individuals with overweight or obesity, instead, high glucose concentrations were observed regardless of the level of perceived stress. Interventions to improve stress management could improve dietary adherence and glycaemic control and could thereby have the potential to improve long-term health and well-being of individuals with type 1 diabetes.
\end{abstract}

\section{Introduction}

Stress is a nonspecific bodily response to any demand made upon it [1]. The detrimental effects of stress are conveyed both directly through neuroendocrine and autonomic responses and indirectly through untoward changes in health behaviours. As adherence to various self-management practices is the cornerstone of daily management of type 1 diabetes, perceived stress could negatively impact the long-term health outcomes of the affected individuals.
Eating is the most frequently reported stress-release method [2]. Importantly, previous research has suggested a change toward unhealthy dietary choices when under stress [3-5], although conflicting results have also been published [6-8]. Studies on the association between stress and eating behaviour in adult individuals with type 1 diabetes are scarce. Over the numerous contacts with healthcare personnel and at diabetes management trainings, the importance of diet on glycaemic control and long-term health is highlighted to individuals with type 1 diabetes. It is therefore likely that 
these individuals are highly aware of the role that diet plays in their daily management. Stressful life events are, however, prone to happen. It would therefore be important to identify how perceived stress impacts the dietary choices, as well as the blood glucose levels, of individuals with type 1 diabetes.

Our aim was therefore to explore whether there are associations between perceived stress and adherence to dietary recommendations in individuals with type 1 diabetes. We also investigated whether perceived stress is associated with blood glucose concentrations and whether a potential association between perceived stress and diet adherence and glycaemic control differs in lean and overweight or obese participants.

\section{Materials and Methods}

Study subjects were participants in the ongoing nationwide Finnish Diabetic Nephropathy (FinnDiane) Study. The aim of the FinnDiane Study is to identify risk factors for diabetes complications in adult individuals with type 1 diabetes. Type 1 diabetes was defined as diabetes onset before the age of 40 years and permanent insulin treatment initiated within a year from the diagnosis. The Ethics Committee of the Helsinki and Uusimaa Hospital District approved the study protocol. The study was carried out in accordance with the Declaration of Helsinki and its later amendments. Written informed consent was obtained from all individuals prior to study participation.

2.1. Clinical Measurements. The procedures conducted at the FinnDiane Study visit have been previously reported [9]. In short, participants' height and weight were measured in light clothing. These measurements were used to calculate body mass index $\left(\mathrm{BMI} ; \mathrm{kg} / \mathrm{m}^{2}\right)$. Glycated haemoglobin $\left(\mathrm{HbA}_{1 \mathrm{c}}\right)$ was measured locally using a standardized assay. Blood was drawn for subsequent analyses of serum lipid, lipoprotein, and creatinine concentrations at the laboratory of the Helsinki University Hospital. The serum creatinine concentration was used to calculate the estimated glomerular filtration rate (eGFR; $\mathrm{ml} / \mathrm{min} / 1.73 \mathrm{~m}^{2}$ ) [10]. The insulin dose was selfreported, and the reported value was used to calculate the dose per body weight (IU/kg).

2.2. Perceived Stress. At the study visit, participants were asked to complete a number of questionnaires. Of interest to the current study, a subset of the FinnDiane Study participants filled out Cohen's 14-item perceived stress scale (PSS) [11]. Using a 5-point Likert scale $(0=$ never, $1=$ almost never, $2=$ sometimes, 3 = fairly often, and $4=$ very often), this questionnaire assesses the extent to which the respondent considers life situations as stressful. After reversing the scores of the positive items, a total score for each individual was obtained by summing up the responses to all the 14 individual items. The total perceived stress score ranges between 0 and 56, with higher scores suggesting higher perceived stress. In the current analyses, the total score was used as a continuous variable. In addition, using a quartile division, we formed three groups. Here, those in the $1^{\text {st }}$ PSS score quartile had low levels of perceived stress; those in the $2^{\text {nd }}$ and $3^{\text {rd }}$ quartiles had moderate levels of perceived stress; and those in the $4^{\text {th }}$ quartile had high levels of perceived stress.
2.3. Dietary Intake. Dietary intake was measured with a validated diet questionnaire [12]. In this questionnaire, participants reported their habitual consumption of coffee, tea, liquid milk products, bread, spreads, and cooking fats. Two questions were formulated to assess the habits of salt consumption. Included was also a food frequency questionnaire, where the frequencies of consuming fish dishes, meat dishes, poultry, sausages and cold cuts, legumes, fresh vegetables, cooked vegetables, potatoes, pasta and rice, fruits and berries, high-fat cheese, low-fat cheese, yoghurt and curd, ice cream, soft drinks, sweet pastry, sweets and chocolate, and fried and grilled foods were queried. Here, the frequencies were reported on a 7-level scale (several times a day, once a day, 4-6 times a week, 2-3 times a week, once a week, 1-3 times a month, and less often or never). From the obtained data, we calculated a diet score for each participant. The bases for calculating the diet score have been previously described in detail [13]. In short, each question of the diet questionnaire was compared against the dietary recommendations. For all the food items for which a distinct recommendation was found, participants were scored based on the level of adherence to the recommendation. In all, 11 such food items were identified from the diet questionnaire, and for each of these items, a score ranging from 0 to 2 was given. The total diet score thus ranged between 0 and 22, with the highest scores denoting closer adherence to the recommendations. The total diet score was used as a continuous variable in the analyses. In addition, the individual scores of the 11 food items were also examined. Participants were also asked to complete a three-day food record twice [14]. The recordings were performed with a 10 -week interval. In the same record, participants also reported their blood glucose measurements. The mean of these measurements was calculated for each participant and used as a continuous variable in the analyses.

2.4. Physical Activity. Participants reported their physical activity in a questionnaire as previously described [15]. In short, for the preceding 12 months, the frequencies, durations, and intensities of the 21 most common types of leisure-time physical activities were reported. For each activity, we calculated an intensity-specific metabolic equivalent of task (MET) as hours per week and summed up all individual MET hours for each participant to obtain the total weekly MET hour. Data on physical activity were used as a covariate in the analyses with the blood glucose concentration as a dependent variable.

2.5. Statistical Analyses. All analyses were conducted with IBM SPSS Statistics for Windows, Version 22.0 (IBM Corp, Armonk, NY, USA), and the alpha value was set to $5 \%$ (two-tailed test). Descriptive data are presented as frequencies for categorical variables and median (interquartile range) for continuous variables with skewed distribution. Unadjusted analyses to examine between-group differences were conducted with the Chi-squared test and Mann-Whitney $U$ test, respectively. Generalized linear regression analysis was used to study the adjusted associations between perceived stress (independent variable) and adherence to the dietary recommendations and blood glucose measurements (dependent 
TABLE 1: Basic characteristics divided by perceived stress.

\begin{tabular}{|c|c|c|c|}
\hline & $\begin{array}{c}\text { Low stress } \\
n=24\end{array}$ & $\begin{array}{l}\text { Moderate stress } \\
\quad n=51\end{array}$ & $\begin{array}{l}\text { High stress } \\
n=25\end{array}$ \\
\hline Stress score & $11(7-12)$ & $19(17-22)^{* * *}$ & $37(30-41)^{* * *}$ \\
\hline Men (\%) & 50.0 & 60.8 & 32.0 \\
\hline Age (years) & $41(38-45)$ & $40(33-46)^{*}$ & $35(31-43)^{*}$ \\
\hline BMI $\left(\mathrm{kg} / \mathrm{m}^{2}\right)$ & $26.6(25.1-29.0)$ & $25.1(23.3-29.9)$ & $26.3(23.3-30.4)$ \\
\hline eGFR (ml/min/1.73 m²) & $107(91-113)$ & $109(97-119)^{*}$ & $115(107-119)^{*}$ \\
\hline $\mathrm{HbA}_{1 \mathrm{c}}(\mathrm{mmol} / \mathrm{mol})$ & $54(51-67)$ & $64(55-69)$ & $59(53-65)$ \\
\hline $\mathrm{HbA}_{1 \mathrm{c}}(\%)$ & $7.1(6.8-8.3)$ & $8.0(7.2-8.5)$ & $7.5(7.0-8.1)$ \\
\hline Cholesterol (mmol/l) & $4.49(3.94-5.03)$ & $4.35(3.95-4.92)$ & $4.51(4.06-5.24)$ \\
\hline HDL-cholesterol (mmol/l) & $1.23(1.00-1.52)$ & $1.42(1.15-1.76)$ & $1.40(1.16-1.76)$ \\
\hline Triglycerides (mmol/l) & $1.0(0.8-1.4)$ & $1.0(0.7-1.6)$ & $0.8(0.7-1.6)$ \\
\hline
\end{tabular}

Data are presented as the median (interquartile range) for continuous variables as they had skewed distribution and frequency for categorical variables. Between-group comparisons were conducted with the Mann-Whitney $U$ test and Chi-squared test, respectively. In the comparisons, the moderate and high perceived stress groups were compared to the group with low perceived stress. Low stress: $1^{\text {st }}$ quartile of the perceived stress scale; moderate stress: $2^{\text {nd }}$ and $3^{\text {rd }}$ quartiles of the perceived stress scale; high stress: $4^{\text {th }}$ quartile of the perceived stress scale; BMI: body mass index. ${ }^{*} p<0.05 ;{ }^{* * *} p<0.001$.

TABLE 2: Diet score divided by perceived stress.

\begin{tabular}{|c|c|c|c|}
\hline & $\begin{array}{c}\text { Low stress } \\
n=24\end{array}$ & $\begin{array}{c}\text { Moderate stress } \\
\quad n=51\end{array}$ & $\begin{array}{c}\text { High stress } \\
\quad n=25\end{array}$ \\
\hline Diet score & $12(9-14)$ & $10(9-12)^{*}$ & $9(6.5-10)^{* *}$ \\
\hline \multicolumn{4}{|l|}{ Components of the diet score } \\
\hline Fish & $1(1-2)$ & $1(0-1)^{*}$ & $0.5(0-1)^{*}$ \\
\hline Fresh vegetables & $2(1-2)$ & $1(0-1)^{* *}$ & $1(0-1)^{*}$ \\
\hline Cooked vegetables & $1.5(0-2)$ & $1(0-2)$ & $1(0-2)$ \\
\hline Fruits and berries & $1(0-2)$ & $1(0-1)$ & $0(0-1.5)$ \\
\hline Sweet pastry & $1.5(0-2)$ & $2(1-2)$ & $1(0.5-2)$ \\
\hline Sweets and chocolate & $0.5(0-2)$ & $1(0-2)$ & $0(0-1)$ \\
\hline Soft drinks & $1(0-2)$ & $1(0-2)$ & $1(0-1)$ \\
\hline Low-fat liquid milk products & $0.5(0-2)$ & $0(0-1)$ & $0(0-1)^{*}$ \\
\hline Vegetable oil spreads & $0(0-2)$ & $0(0-2)$ & $0(0-2)$ \\
\hline Vegetable oil cooking fats & $2(2-2)$ & $2(0-2)^{* *}$ & $2(0-2)$ \\
\hline Salt & $0(0-1)$ & $0(0-1)$ & $0(0-1)$ \\
\hline
\end{tabular}

Data are presented as the median (interquartile range). Between-group comparisons were conducted with the Mann-Whitney $U$ test. In the comparisons, the moderate and high perceived stress groups were compared to the group with low perceived stress. Low stress: $1^{\text {st }}$ quartile of the perceived stress scale; moderate: $2^{\text {nd }}$ and $3^{\text {rd }}$ quartiles of the perceived stress scale; high stress: $4^{\text {th }}$ quartile of the perceived stress scale. ${ }^{*} p<0.05 ;{ }^{* *} p<0.01$.

variables). The analyses were first conducted in the whole sample comparing individuals with low perceived stress to those with moderate and high perceived stress. We then investigated the associations between perceived stress and the dependent variables separately in individuals with BMI at the range of normal weight $\left(<25 \mathrm{~kg} / \mathrm{m}^{2}\right)$ and those with overweight or obesity (BMI $\left.\geq 25 \mathrm{~kg} / \mathrm{m}^{2}\right)$.

\section{Results}

3.1. Stress and Dietary Adherence in the Whole Sample. Data were available from 100 individuals (median age 40 years, age range from 25 to 71 years, $51 \%$ men). In all, 24 participants reported low levels of perceived stress, while moderate and high levels of perceived stress were reported by 51 and 25 individuals, respectively (Table 1). Apart from individuals with moderate and high levels of perceived stress being younger and having higher eGFR as compared to those with low perceived stress, the groups were comparable with respect to the basic characteristics.

In the unadjusted analyses, compared to those with low perceived stress, individuals with moderate and high perceived stress scored significantly lower in the diet score and its fish and fresh vegetable components (Table 2). In addition, individuals with moderate levels of perceived stress had lower scores for the adherence to vegetable oil-based cooking fats, while those with high perceived stress had lower scores for the adherence to low-fat liquid milk product recommendations.

After adjusting for age, sex, eGFR, and BMI, those with moderate levels of perceived stress had lower scores in 
TABLE 3: The multivariable association between perceived stress and diet score.

\begin{tabular}{|c|c|c|c|c|c|c|c|c|}
\hline & $\begin{array}{c}\text { Low stress } \\
\text { Mean (95 Wald } \\
\text { CI) }\end{array}$ & $\begin{array}{c}\text { Moderate stress } \\
\text { Mean (95 Wald } \\
\text { CI) }\end{array}$ & $p$ & $\begin{array}{c}\text { Low stress } \\
\text { Mean (95 Wald } \\
\text { CI) }\end{array}$ & $\begin{array}{c}\text { High stress } \\
\text { Mean (95 Wald } \\
\text { CI) }\end{array}$ & $p$ & $\begin{array}{c}\text { Stress score } \\
B(95 \% \text { Wald } \\
\text { CI })\end{array}$ & $p$ \\
\hline Diet score & $11.5(10.3-12.7)$ & $10.0(9.2-10.9)$ & 0.056 & $11.3(10.0-12.5)$ & $9.1(7.8-10.3)$ & 0.016 & $\begin{array}{c}-0.10(-0.16-- \\
0.05)\end{array}$ & 0.001 \\
\hline \multicolumn{9}{|l|}{$\begin{array}{l}\text { Components of the diet } \\
\text { score }\end{array}$} \\
\hline Fish & $1.2(0.9-1.5)$ & $0.8(0.6-1.0)$ & 0.068 & $1.2(0.9-1.5)$ & $0.7(0.4-0.9)$ & 0.018 & $\begin{array}{c}-0.02(-0.03-- \\
0.01)\end{array}$ & 0.029 \\
\hline Fresh vegetables & $1.3(1.0-1.6)$ & $0.8(0.5-1.0)$ & 0.003 & $1.3(1.0-1.6)$ & $0.8(0.5-1.1)$ & 0.010 & $\begin{array}{c}-0.02(-0.03-- \\
0.01)\end{array}$ & 0.021 \\
\hline Cooked vegetables & $1.1(0.8-1.5)$ & $1.0(0.8-1.2)$ & 0.561 & $1.2(0.8-1.6)$ & $1.1(0.7-1.5)$ & 0.761 & $\begin{array}{l}0.00(-0.02- \\
0.02)\end{array}$ & 0.844 \\
\hline Fruits and berries & $0.9(0.6-1.2)$ & $0.7(0.5-0.9)$ & 0.461 & $0.9(0.6-1.3)$ & $0.7(0.4-1.1)$ & 0.467 & $\begin{array}{c}-0.01(-0.02- \\
0.01)\end{array}$ & 0.488 \\
\hline Sweet pastry & $1.2(0.9-1.5)$ & $1.5(1.3-1.7)$ & 0.083 & $1.1(0.8-1.4)$ & $1.2(0.9-1.6)$ & 0.590 & $\begin{array}{c}-0.01(-0.02- \\
0.01)\end{array}$ & 0.622 \\
\hline Sweets and chocolate & $0.9(0.5-1.2)$ & $1.0(0.7-1.2)$ & 0.655 & $0.8(0.4-1.1)$ & $0.8(0.5-1.1)$ & 0.894 & $\begin{array}{c}-0.01(-0.02- \\
0.01)\end{array}$ & 0.403 \\
\hline Soft drinks & $1.0(0.7-1.3)$ & $1.0(0.8-1.2)$ & 0.946 & $1.0(0.7-1.3)$ & $0.9(0.6-1.2)$ & 0.692 & $\begin{array}{c}0.00(-0.01- \\
0.02)\end{array}$ & 0.922 \\
\hline $\begin{array}{l}\text { Low-fat liquid milk } \\
\text { products }\end{array}$ & $0.8(0.5-1.1)$ & $0.5(0.3-0.7)$ & 0.143 & $0.8(0.5-1.1)$ & $0.3(0.0-0.6)$ & 0.014 & $\begin{array}{c}-0.02(-0.03-- \\
0.01)\end{array}$ & 0.012 \\
\hline Vegetable oil spreads & $0.8(0.4-1.2)$ & $0.9(0.6-1.2)$ & 0.683 & $0.7(0.3-1.1)$ & $0.8(0.4-1.2)$ & 0.866 & $\begin{array}{c}-0.01(-0.02- \\
0.01)\end{array}$ & 0.593 \\
\hline $\begin{array}{l}\text { Vegetable oil cooking } \\
\text { fats }\end{array}$ & $1.8(1.4-2.1)$ & $1.5(1.3-1.7)$ & 0.220 & $1.8(1.5-2.1)$ & $1.3(1.0-1.6)$ & 0.033 & $\begin{array}{c}-0.03(-0.04-- \\
0.01)\end{array}$ & 0.001 \\
\hline Salt & $0.6(0.3-0.9)$ & $0.4(0.2-0.6)$ & 0.366 & $0.5(0.3-0.8)$ & $0.4(0.2-0.7)$ & 0.674 & $\begin{array}{c}-0.01(-0.02- \\
0.01)\end{array}$ & 0.476 \\
\hline
\end{tabular}

Generalized linear regression analysis. CI: confidence interval. All models are adjusted for age, sex, estimated glomerular filtration rate, and BMI. Low stress: $1^{\text {st }}$ quartile of the perceived stress scale; moderate stress: $2^{\text {nd }}$ and $3^{\text {rd }}$ quartiles of the perceived stress scale; high stress: $4^{\text {th }}$ quartile of the perceived stress scale.

adhering to the fresh vegetable recommendations compared to individuals with low perceived stress (Table 3). Instead, those with high perceived stress had a significantly lower diet score and lower scores for its fish, fresh vegetable, low-fat liquid milk product, and vegetable oil-based cooking fat components. After the same adjustments, a higher stress score as a continuous variable was associated with a lower diet score and lower adherence to the recommendations related to the consumption of fish, fresh vegetables, low-fat liquid milk products, and vegetable oil-based cooking fats.

3.2. Stress and Dietary Adherence in the BMI Categories. We then investigated whether the association between stress and dietary adherence was modified by being overweight or obese. Compared to the normal-weight participants, individuals with overweight or obesity had comparable perceived stress scores but were older and had lower HDL-cholesterol concentration and higher triglyceride concentration (Table 4).

In the age-, sex-, and eGFR-adjusted models, individuals both with normal weight and overweight or obesity exhibited a negative association between the stress score and the diet score (Table 5). However, the groups divided by BMI were different with respect to the individual components of the diet score that were associated with the stress score. Unlike in those with normal weight, in individuals with overweight or obesity, the perceived stress score was associated with lower adherence to fish and fresh vegetable recommendations. Instead, only those with normal weight exhibited lower adherence to low-fat liquid milk product recommendations with increasing levels of perceived stress. Both BMI groups, however, showed a negative association between adherence to vegetable oil-based cooking fat recommendations and the stress score.

3.3. Stress and Blood Glucose Measurements in the Whole Sample. Altogether, 71 participants had reported their blood glucose measurements in a diary. Adjusted for age, sex, eGFR, BMI, physical activity, and insulin dose, individuals with low and moderate levels of perceived stress were comparable with respect to the mean reported blood glucose concentrations $(8.0 \mathrm{mmol} / \mathrm{l}(95 \%$ Wald confidence interval $7.3-$ $8.7 \mathrm{mmol} / \mathrm{l})$ vs. $8.7 \mathrm{mmol} / \mathrm{l}(8.2-9.2 \mathrm{mmol} / \mathrm{l})$, respectively, $p=$ 0.131 ). Comparison between individuals with low and high perceived stress yielded similar results $(8.0 \mathrm{mmol} / \mathrm{l}$ (7.4$8.7 \mathrm{mmol} / \mathrm{l})$ vs. $8.3 \mathrm{mmol} / \mathrm{l}(7.6-9.0 \mathrm{mmol} / \mathrm{l})$, respectively, $p=$ $0.575)$. 
TABLE 4: Basic characteristics divided by BMI classification.

\begin{tabular}{|c|c|c|c|}
\hline & $\begin{array}{c}\mathrm{BMI}<25 \mathrm{~kg} / \mathrm{m}^{2} \\
n=42\end{array}$ & $\begin{array}{c}\mathrm{BMI} \geq 25 \mathrm{~kg} / \mathrm{m}^{2} \\
n=58\end{array}$ & $p$ \\
\hline Stress score & $21(17-27)$ & $17(13-25)$ & 0.212 \\
\hline Men (\%) & 47.6 & 53.4 & 0.686 \\
\hline Age (years) & $37(31-43)$ & $41(37-45)$ & 0.047 \\
\hline BMI $\left(\mathrm{kg} / \mathrm{m}^{2}\right)$ & $23.3(21.8-24.5)$ & $28.4(26.7-31.7)$ & $<0.001$ \\
\hline $\mathrm{eGFR}\left(\mathrm{ml} / \mathrm{min} / 1.73 \mathrm{~m}^{2}\right)$ & $111(102-118)$ & $109(93-117)$ & 0.194 \\
\hline $\mathrm{HbA}_{1 \mathrm{c}}(\mathrm{mmol} / \mathrm{mol})$ & $57(52-67)$ & $63(54-67)$ & 0.170 \\
\hline $\mathrm{HbA}_{1 \mathrm{c}}(\%)$ & $7.4(6.9-8.3)$ & $7.9(7.1-8.3)$ & 0.170 \\
\hline Cholesterol (mmol/l) & $4.36(4.02-4.60)$ & $4.54(3.99-5.12)$ & 0.271 \\
\hline HDL-cholesterol (mmol/l) & $1.49(1.27-1.87)$ & $1.27(1.03-1.58)$ & 0.018 \\
\hline Triglycerides (mmol/l) & $0.8(0.7-1.1)$ & $1.1(0.8-2.0)$ & 0.002 \\
\hline
\end{tabular}

Data are presented as the median (interquartile range) for continuous variables as they had skewed distribution and frequency for categorical variables. Between-group comparisons were conducted with the Mann-Whitney $U$ test and Chi-squared test, respectively. BMI: body mass index.

TABLE 5: The multivariable association between perceived stress score and diet score divided by BMI classification.

\begin{tabular}{|c|c|c|c|c|}
\hline & $\begin{array}{c}\mathrm{BMI}<25 \mathrm{~kg} / \mathrm{m}^{2} \\
B(95 \% \text { Wald CI })\end{array}$ & $p$ & $\begin{array}{c}\mathrm{BMI} \geq 25 \mathrm{~kg} / \mathrm{m}^{2} \\
B(95 \% \text { Wald CI })\end{array}$ & $p$ \\
\hline Diet score & $-0.17(-0.27--0.06)$ & 0.002 & $-0.07(-0.14--0.01)$ & 0.022 \\
\hline \multicolumn{5}{|l|}{ Components of the diet score } \\
\hline Fish & $0.01(-0.03-0.03)$ & 0.760 & $-0.02(-0.04--0.01)$ & 0.002 \\
\hline Fresh vegetables & $-0.01(-0.04-0.02)$ & 0.373 & $-0.02(-0.04--0.01)$ & 0.023 \\
\hline Cooked vegetables & $0.00(-0.03-0.03)$ & 0.985 & $0.00(-0.02-0.02)$ & 0.880 \\
\hline Fruits and berries & $0.00(-0.02-0.03)$ & 0.949 & $-0.01(-0.03-0.01)$ & 0.341 \\
\hline Sweet pastry & $-0.01(-0.04-0.01)$ & 0.303 & $0.00(-0.02-0.02)$ & 0.964 \\
\hline Sweets and chocolate & $-0.02(-0.05-0.01)$ & 0.115 & $0.00(-0.02-0.02)$ & 0.938 \\
\hline Soft drinks & $-0.02(-0.04-0.00)$ & 0.089 & $0.01(-0.01-0.03)$ & 0.326 \\
\hline Low-fat liquid milk products & $-0.02(-0.05--0.01)$ & 0.043 & $-0.02(-0.03-0.00)$ & 0.088 \\
\hline Vegetable oil spreads & $-0.03(-0.06-0.00)$ & 0.071 & $0.01(-0.02-0.03)$ & 0.657 \\
\hline Vegetable oil cooking fats & $-0.03(-0.06--0.01)$ & 0.039 & $-0.02(-0.04--0.01)$ & 0.010 \\
\hline Salt & $-0.02(-0.04-0.00)$ & 0.053 & $0.00(-0.01-0.02)$ & 0.663 \\
\hline
\end{tabular}

Generalized linear regression analysis. BMI: body mass index; CI: confidence interval. All models are adjusted for age, sex, and estimated glomerular filtration rate.

3.4. Stress and Blood Glucose Measurements in the BMI Categories. In all, 31 individuals with normal weight and 40 individuals with overweight or obesity had diary data on the blood glucose concentrations. In normal-weight participants, compared to those with low perceived stress, individuals with moderate perceived stress had significantly higher mean blood glucose concentrations $(6.3 \mathrm{mmol} / \mathrm{l}$ (4.7$7.9 \mathrm{mmol} / \mathrm{l})$ vs. $9.1 \mathrm{mmol} / \mathrm{l}(8.4-9.9 \mathrm{mmol} / \mathrm{l})$, respectively, $p=$ 0.002), after adjustments for age, sex, eGFR, physical activity, and insulin dose. Similarly, significant differences in blood glucose concentrations were observed in lean individuals between those with low and high perceived stress $(6.5 \mathrm{mmol} / \mathrm{l}$ $(5.6-7.5 \mathrm{mmol} / \mathrm{l})$ vs. $8.4 \mathrm{mmol} / \mathrm{l}(7.8-9.0 \mathrm{mmol} / \mathrm{l})$, respectively, $p=0.004)$. In those with overweight or obesity, instead, the level of glycaemia was no different between those with low and moderate perceived stress $(8.4 \mathrm{mmol} / \mathrm{l}(7.7-9.0 \mathrm{mmol} / \mathrm{l})$ vs. $8.3 \mathrm{mmol} / \mathrm{l}(7.7-8.8 \mathrm{mmol} / \mathrm{l})$, respectively, $p=0.780)$ and between those with low and high perceived stress $(8.6 \mathrm{mmol} / \mathrm{l}$
(8.0-9.3 mmol/l) vs. $7.9 \mathrm{mmol} / \mathrm{l}(7.0-8.8 \mathrm{mmol} / \mathrm{l})$, respectively, $p=0.262)$.

\section{Discussion}

In the current study, high perceived stress was significantly associated with less healthy diet judged by lower adherence to the dietary recommendations. In particular, the scores reflecting the level of adherence to fish, fresh vegetable, lowfat liquid milk products, and vegetable oil-based cooking fat recommendations were lower in individuals reporting high levels of stress. We also investigated whether the association between stress and dietary adherence would be modified by overweight or obesity. While stress was observed to reduce the adherence to the overall dietary recommendations in both lean and those with corpulence, there seemed to be some differences in which individual recommendations were affected in these two groups. Notably, unlike in lean individuals, stress 
was associated with reduced adherence to fish and fresh vegetable recommendations in those with overweight or obesity. Finally, we investigated the association between perceived stress and glycaemia. Here, stress was associated with higher levels of mean blood glucose levels only in lean participants. In individuals with overweight or obesity, instead, high glucose concentrations were observed regardless of the level of perceived stress.

While we are not aware of other studies on perceived stress and adherence to the dietary recommendations in adults with type 1 diabetes, the association has been investigated in a number of other populations. Among these is the study in 5077 adults by Isasi et al., who observed an inverse association between perceived stress and a healthy eating index suggesting a less healthy eating pattern among those reporting higher perceived stress during the past month [3]. Similarly, among 1189 university students in Finland, perceived stress was negatively associated with a dietary guideline adherence index [4]. Moreover, compared to the normal-weight students, this association was more pronounced in those with overweight. An inverse relationship between the level of perceived stress and the overall diet quality was also observed in a sample of European adolescents [5]. In addition to the above-mentioned observations showing an adverse association between stress and diet quality, a number of studies with no association have also been published. Among these is the study by Carson et al., who investigated the relationship between perceived stress and meeting of food group recommendations in 355 African American women [6]. In their study, neither stress score nor grouping of participants based on the median stress score was associated with meeting the recommendations. Similarly, in a large sample of university students in Egypt, no association was observed between stress and an index of dietary guideline adherence [7]. In a sample of overweight and obese working-age Finns, again, the adherence to a healthy diet was no different among the tertiles of perceived stress [8]. However, a higher level of perceived stress was associated with lower intuitive eating, lower eating competence, and lower cognitive restraints. Moreover, perceived stress was positively associated with uncontrolled and emotional eating and a higher tendency to use food as a reward.

In addition to the studies investigating the association between perceived stress and adherence to the overall dietary recommendations, a number of studies have also reported the association between stress and individual food items. Here, similar to the observations made in the current study, perceived stress was associated with reduced consumption of fruits and vegetables in some studies [7, 16, 17] but not all [18]. A number of studies have also highlighted the positive association between perceived stress and consumption of sweets [17-19]. However, in line with the observations by El Ansari et al. [4, 7], no such association was evident in the current study. This lack of association between stress and the consumption of sweet food items, in the current study, may partly be explained by the population in question. After all, the importance of avoiding sweet foods in controlling blood glucose fluctuations in type 1 diabetes is likely acknowledged regardless of the participants' stress level. Yet, a number of studies have linked perceived stress with higher intake of fast foods or snacks $[16-18,20]$. Preference for quickly available meals when stressed is in concordance with the reports that perceived stress is also associated with haphazard meal planning [21]. During stressful periods, individual may, therefore, be less likely to plan one's meals carefully. While "fast foods," as an individual food item, were not included in our questionnaire, we did observe that perceived stress was associated with lower adherence to the recommendations related to fish, fresh vegetables, low-fat liquid milk products, and vegetable oil-based cooking fats, that is, food items that may be related to properly planned meals. Of interest, the association between perceived stress and haphazard meal planning has shown to be particularly pronounced in individuals with overweight or obesity [21]. In line with these observations, when we divided the study population by the BMI status, perceived stress was associated with reduced adherence with fish and fresh vegetable recommendations only in subjects with overweight or obesity.

Using functional magnetic resonance, Tyron et al. observed that, in participants reporting higher chronic stress, pictures of foods high in energy content elicited exaggerated activity in regions of the brain involving reward, motivation, and habitual decision-making [22]. Moreover, viewing the same pictures of high-energy foods was associated with significant deactivation of frontal brain regions that are linked to strategic planning and emotional control. These results suggest that stress may alter the brain's response to foods in ways that predispose individuals to unhealthy dietary choices. While poor stress management has been associated with worse health risk behaviour profile, it is noteworthy that health behaviour change intervention targeted to improve stress management skills has also been associated with improvements in health behaviours [23].

Stress may impair glucose metabolism through dysregulation of the hypothalamic-pituitary-adrenal and sympatheticadrenal-medullary axes [24]. Indeed, acute stress activates central, autonomic, neuroendocrine, and immune systems in order to harness the individual with sufficient fuel to either fight or flight in the face of the stressor. In such a state, insulin resistance also occurs. Subsequently, it would be reasonable to expect increased blood glucose levels upon experiencing stress. However, in the current study, perceived stress was not in the whole population associated with the daily mean of the blood glucose measurements. In line with our observations, Halford et al. reported that only 7 out of 15 patients exhibited increased blood glucose levels when stressed and concluded that stress influenced blood glucose levels at least in some individuals with diabetes [25]. Similarly, Kramer et al. encountered major heterogeneity in the association between stress and blood glucose concentrations in individuals with diabetes, as less than half of the participants showed any such association [26]. Of interest, when investigating the association between stress and glycaemia, Ozaslan et al. noted that the blood glucose-rising effect of stress was diminished by increasing BMI [27]. Our observations with higher blood glucose concentrations in lean subjects reporting stress and no association between perceived stress and glycaemia in overweight or obese subjects are in line with the reports by Ozaslan et al., suggesting that additional factors impact glucose dynamics under stress. Indeed, prevailing 
insulin resistance, related to obesity, may mask the association between perceived stress and glycaemia [28].

Well-defined study participants with objectively measured height and weight are important strengths of this study. The study population was, however, small. Due to the small sample size, we could not, for example, investigate the sex differences in the perceived stress and dietary adherence. This may be important, as $68 \%$ of those reporting high levels of stress were women, compared to $50 \%$ of those with low perceived stress. Larger studies are needed to further elaborate such potential differences. Another limitation is the use of self-reported methods to study dietary intake, blood glucose values, and stress. Importantly, incorporating a physiologic measurement of stress, such as measuring salivary cortisol, would have reduced the measurement error related to subjective assessment. Finally, due to the observational nature of the study, no causal relationships can be determined.

\section{Conclusions}

Perceived stress was associated with reduced adherence to dietary recommendations in participants with type 1 diabetes: fish, fresh vegetable, low-fat liquid milk product, and vegetable oil-based cooking fat recommendations, in particular. Moreover, while in overweight or obese individuals, high blood glucose concentrations were observed regardless of the stress level; stress perception in lean participants was observed to impair the glycaemic control. Interventions to improve stress management could improve dietary adherence and glycaemic control and could thereby have the potential to improve long-term health and well-being of individuals with type 1 diabetes.

\section{Data Availability}

The data used to support the findings of this study are available from the corresponding authors upon request.

\section{Disclosure}

Funding agencies did not contribute to the study design, conduct of the study, analysis of samples or data, interpretation of the findings, writing of the manuscript, or in the decision to submit the manuscript for publication.

\section{Conflicts of Interest}

Professor Per-Henrik Groop has received investigatorinitiated grants from Eli Lilly and Roche and is an advisory board member for AbbVie, Astellas, Astra Zeneca, Bayer, Boehringer-Ingelheim, Eli Lilly, Janssen, Medscape, MSD, Mundipharma, Novartis, Novo Nordisk, and Sanofi. He has received lecture honoraria from Astellas, Astra Zeneca, Boehringer-Ingelheim, Eli Lilly, Elo Water, Genzyme, MSD, Novartis, Novo Nordisk, PeerVoice, Sanofi, and SCIARC. All other authors declare no conflicts of interest.

\section{Authors' Contributions}

All authors took part in designing the study. A.J.A. analysed the data and wrote the first draft of the paper. A.J.A. and P.H.G. had the primary responsibility for the final content. All authors have contributed to the writing and approved the final manuscript.

\section{Acknowledgments}

This study was supported by grants from the Academy of Finland (grant number 316664), Novo Nordisk Foundation (\#NNF OC0013659), Signe ja Ane Gyllenbergin Säätiö, Folkhälsan Research Foundation, Helsinki University Hospital Research Funds (TYH2018207), Wilhelm och Else Stockmanns Stiftelse, Liv och Hälsa Society, Finnish Society of Medicine (Finska Läkaresällskapet), and Päivikki ja Sakari Sohlbergin Säätiö. The skilled technical assistance of Anna Sandelin, Mira Korolainen, and Jaana Tuomikangas is gratefully acknowledged. The authors also acknowledge all the physicians and nurses at each centre participating in the recruitment of patients (supplementary file).

\section{Supplementary Materials}

List of physicians and nurses participating in the enrollment of patients. (Supplementary materials)

\section{References}

[1] H. Selye, Stress without Distress, J. B. Lippencott Comp, New York, NY, USA, 1974.

[2] E. S. Nahm, J. Warren, S. Zhu, M. An, and J. Brown, "Nurses' self-care behaviors related to weight and stress," Nursing Outlook, vol. 60, no. 5, pp. e23-e31, 2012.

[3] C. R. Isasi, C. M. Parrinello, M. M. Jung et al., "Psychosocial stress is associated with obesity and diet quality in Hispanic/Latino adults," Annals of Epidemiology, vol. 25, no. 2, pp. 84-89, 2015.

[4] W. El Ansari, S. Suominen, and G. Berg-Beckhoff, "Mood and food at the University of Turku in Finland: nutritional correlates of perceived stress are most pronounced among overweight students," International Journal of Public Health, vol. 60, no. 6, pp. 707-716, 2015.

[5] T. De Vriendt, E. Clays, I. Huybrechts et al., "European adolescents' level of perceived stress is inversely related to their diet quality: the Healthy Lifestyle in Europe by Nutrition in Adolescence study," The British Journal of Nutrition, vol. 108, no. 2, pp. 371-380, 2012.

[6] T. L. Carson, R. Desmond, S. Hardy et al., "A study of the relationship between food group recommendations and perceived stress: findings from black women in the Deep South," Journal of Obesity, vol. 2015, Article ID 203164, 7 pages, 2015.

[7] W. El Ansari and G. Berg-Beckhoff, "Nutritional correlates of perceived stress among university students in Egypt," International Journal of Environmental Research and Public Health, vol. 12, no. 11, pp. 14164-14176, 2015.

[8] E. Järvelä-Reijonen, L. Karhunen, E. Sairanen et al., "High perceived stress is associated with unfavorable eating behavior in overweight and obese Finns of working age," Appetite, vol. 103, pp. 249-258, 2016. 
[9] A. J. Ahola, L. M. Thorn, M. Saraheimo, C. Forsblom, P.H. Groop, and On behalf of the Finndiane Study Group, "Depression is associated with the metabolic syndrome among patients with type 1 diabetes," Annals of Medicine, vol. 42, no. 7, pp. 495-501, 2010.

[10] A. S. Levey, L. A. Stevens, C. H. Schmid et al., "A new equation to estimate glomerular filtration rate," Annals of Internal Medicine, vol. 150, no. 9, pp. 604-612, 2009.

[11] S. Cohen, T. Kamarck, and R. Mermelstein, "A global measure of perceived stress," Journal of Health and Social Behavior, vol. 24, no. 4, pp. 385-396, 1983.

[12] A. J. Ahola, M. I. Lassenius, C. Forsblom, V. Harjutsalo, M. Lehto, and P. H. Groop, "Dietary patterns reflecting healthy food choices are associated with lower serum LPS activity," Scientific Reports, vol. 7, no. 1, p. 6511, 2017.

[13] A. J. Ahola, V. Mikkilä, M. Saraheimo et al., "Sense of coherence, food selection and leisure time physical activity in type 1 diabetes," Scandinavian Journal of Public Health, vol. 40, no. 7, pp. 621-628, 2012.

[14] A. J. Ahola, V. Mikkilä, S. Mäkimattila et al., "Energy and nutrient intakes and adherence to dietary guidelines among Finnish adults with type 1 diabetes," Annals of Medicine, vol. 44, no. 1, pp. 73-81, 2011.

[15] J. Waden, H. Tikkanen, C. Forsblom et al., "Leisure time physical activity is associated with poor glycemic control in type 1 diabetic women: the FinnDiane study," Diabetes Care, vol. 28, no. 4, pp. 777-782, 2005.

[16] M. Cartwright, J. Wardle, N. Steggles, A. E. Simon, H. Croker, and M. J. Jarvis, "Stress and dietary practices in adolescents," Health Psychology, vol. 22, no. 4, pp. 362-369, 2003.

[17] K. D. Laugero, L. M. Falcon, and K. L. Tucker, "Relationship between perceived stress and dietary and activity patterns in older adults participating in the Boston Puerto Rican Health Study," Appetite, vol. 56, no. 1, pp. 194-204, 2011.

[18] V. L. Errisuriz, K. E. Pasch, and C. L. Perry, "Perceived stress and dietary choices: the moderating role of stress management," Eating Behaviors, vol. 22, pp. 211-216, 2016.

[19] Y. Kim, H. Y. Yang, A. J. Kim, and Y. Lim, “Academic stress levels were positively associated with sweet food consumption among Korean high-school students," Nutrition, vol. 29, no. 1, pp. 213-218, 2013.

[20] L. M. Groesz, S. McCoy, J. Carl et al., "What is eating you? Stress and the drive to eat," Appetite, vol. 58, no. 2, pp. 717721, 2012

[21] R. Sims, S. Gordon, W. Garcia et al., "Perceived stress and eating behaviors in a community-based sample of African Americans," Eating Behaviors, vol. 9, no. 2, pp. 137-142, 2008.

[22] M. S. Tryon, C. S. Carter, R. Decant, and K. D. Laugero, "Chronic stress exposure may affect the brain's response to high calorie food cues and predispose to obesogenic eating habits," Physiology \& Behavior, vol. 120, pp. 233-242, 2013.

[23] J. M. Lipschitz, A. L. Paiva, C. A. Redding, S. Butterworth, and J. O. Prochaska, "Co-occurrence and coaction of stress management with other health risk behaviors," Journal of Health Psychology, vol. 20, no. 7, pp. 1002-1012, 2013.

[24] I. Kyrou and C. Tsigos, "Stress hormones: physiological stress and regulation of metabolism," Current Opinion in Pharmacology, vol. 9, no. 6, pp. 787-793, 2009.

[25] W. K. Halford, S. Cuddihy, and R. H. Mortimer, "Psychological stress and blood glucose regulation in type I diabetic patients," Health Psychology, vol. 9, no. 5, pp. 516-528, 1990.
[26] J. R. Kramer, J. Ledolter, G. N. Manos, and M. L. Bayless, "Stress and metabolic control in diabetes mellitus: methodological issues and an illustrative analysis," Annals of Behavioral Medicine, vol. 22, no. 1, pp. 17-28, 2000.

[27] B. Ozaslan, S. D. Patek, J. H. Grabman et al., "Body mass index effect on differing responses to psychological stress in blood glucose dynamics in patients with type 1 diabetes," Journal of Diabetes Science and Technology, vol. 12, no. 3, pp. 657-664, 2018.

[28] B. B. Kahn and J. S. Flier, "Obesity and insulin resistance," The Journal of Clinical Investigation, vol. 106, no. 4, pp. 473-481, 2000. 